\title{
Assessing the long-term outcomes of bariatric surgery in adolescents
}

...patients who underwent

bariatric

surgery during

adolescence

experienced

significant

and sustained

weight loss...

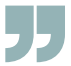

Obesity, and its associated comorbidities, is increasingly prevalent in adolescents. Whereas the use of bariatric surgery in adults with obesity is fairly well established, long-term data on the use of this procedure in adolescents have been lacking. Now, two studies published in the Lancet Diabetes \& Endocrinology report long-term follow-up data from two cohorts of adolescents with severe obesity who underwent Roux-en-Y gastric bypass.

In the first study, Thomas Inge and colleagues identified 58 patients with severe obesity $\left(\mathrm{BMI}>40 \mathrm{~kg} / \mathrm{m}^{2}\right)$ who underwent Roux-en-Y gastric bypass when they were 13-21 years old at the Cincinnati Children's Hospital Medical Center, Ohio, USA. At a mean follow-up of 8 years after surgery, all patients had a substantial and sustained weight loss ( $\sim 30 \%$ of initial body weight). The researchers also found significant declines in elevated blood pressure, dyslipidaemia and type 2 diabetes mellitus. However, mild anaemia was found in $46 \%$ of patients, hyperparathyroidism in $45 \%$ and low levels of vitamin $\mathrm{B} 12$ in $16 \%$.

"An important finding was that individuals who underwent the operation early after the diagnosis of severe obesity were more likely to achieve a more normal weight over the long term compared with those who underwent the operation after developing more-severe forms of obesity," explains Inge. "These findings have practical implications for thinking about the timing of bariatric surgery during progressive weight gain in adolescence”.

The authors note that long-term follow-up of adolescent patients who undergo Roux-en-Y gastric bypass should focus on ensuring adherence to dietary supplements and monitoring for micronutrient deficiencies. "Future research in this area should focus on outcomes of other modern operations, such as vertical sleeve gastrectomy," says Inge.

The second study included 5-year follow-up of 81 adolescent patients with severe obesity who underwent laparascopic Rouxen-Y gastric bypass at one of three specialised centres in Sweden. The study also included data from 80 adolescent controls undergoing conservative treatment and 81 adult controls who underwent Roux-en-Y gastric bypass.

As in the first study, patients who underwent bariatric surgery during adolescence experienced significant and sustained weight loss, although many still had a BMI in the overweight or obese categories. These results were similar to those of the adults who had bariatric surgery and were better than those of the control adolescents. Bariatric surgery during adolescence also resulted in improvements in comorbidities and cardiovascular risk factors. However, $25 \%$ of these patients underwent additional surgery for complications of the Roux-en-Y procedure or rapid weight loss, and $72 \%$ had some kind of nutritional deficiency at follow-up.

Torsten Olbers, corresponding author of the second paper, concludes that the positive effects of bariatric surgery in these patients outweigh the risk of negative effects and complications. "In Sweden, we are discussing formal integration of bariatric surgery in programmes for treatment of severe obesity in postpubertal adolescents," says Olbers.

Claire Greenhill

ORIGINAL ARTICLES Inge, T. H. et al. Long-term outcomes of bariatric surgery in adolescents with severe obesity (FABS-5+): a prospective follow-up analysis. Lancet Diabetes Endocrinol. http://dx.doi org/10.1016/S2213-8587(16)30315-1 (2017) | Olbers, T. et al. Laparoscopic Roux-en-Y gastric bypass in adolescents with severe obesity (AMOS): a prospective, 5-year, Swedish nationwide study. Lancet Diabetes Endocrinol. http://dx.doi. org/10.1016/S2213-8587(16)30424-7 (2017) FURTHER READING Frühbeck, G. Bariatric and metabolic surgery: a shift in eligibility and success criteria. Nat. Rev. Endocrinol. 11, 465-477 (2015) 